\title{
Improved home care activities for brain- injured children: the creation of a wireless platform
}

\author{
A. Tura ${ }^{1}$, L. Quareni ${ }^{2}$, D. Longo ${ }^{2} \&$ M. Badanai ${ }^{2}$ \\ ${ }^{I} I S I B-C N R$, Padova, Italy \\ ${ }^{2}$ QUBIsoft, Padova, Italy
}

\begin{abstract}
A project is presented for creation of a network for managing Home Care activities in brain-injured children. At the patient's side, a Home Care platform is provided made of a medical instrument, and a device for collecting measured data and for accessing the Web portal of the project. The medical instrument is wearable for maximum patient's freedom, and transmits the measured physiological data (blood oxygen saturation, heart rate and respiration rate) to the collecting device through an innovative radio communication technology called Bluetooth. From the collecting device, the data are transmitted to a Service Center again with wireless radio technology (at the moment GPRS, in future UMTS). The measured patients' data are then available through Internet for all the professionals for Health Care activities involved in the project. Through the Web portal, the patients and their families will directly be in touch with the professional, as well as with associations and other social entities. The final outcomes of the project are reducing hospitalization and corresponding assistance costs, and removing frustration from families making them feel inside and organized and efficient virtual community.
\end{abstract}

\section{Introduction}

The project presented in this communication develops a platform to efficiently and adequately manage Home Care activities in a specific patient population: brain-injured children. This project is carried on by a Consortium of Partners, 
from research, industrial, and no-profit world, and is co-founded by the European Commission (Karma2, IST Project 2001-32320).

Brain-injured children require rehabilitation treatments and long term (non ending) care: they need continuous at-home family assistance. These activities involve many actors:

- all the professionals involved in the health, social and psychological care delivery on one hand;

- the patients, the families and their associations on the other hand.

In the context of long-term assistance activities home care is a need, often much more effective than in-hospital treatments $[1,2]$. In order to reach the objective of giving an adequate support from a clinical, logistic and social point of view, we need to:

- manage all the assistance activities;

- keep in touch, easily and effectively, all the persons involved;

- deliver the appropriate information where needed.

In more detail, Karma2 implements a rational solution through an infrastructure for telemedicine, easily accessible. It mostly establishes an organisational network that coordinates and manages all the persons and activities, delivering pertinent and updated information. Karma2 establishes a structural network connection between (Figure 1):

1. Hospital Specialist (diagnosis and clinical protocols);

2. Logistic organisation (technical and logistic support);

3. Administrative structure (local health organisation, social services);

4. Local medical resources (GP's);

5. Service Company / Organisation (nurses, physiotherapists).

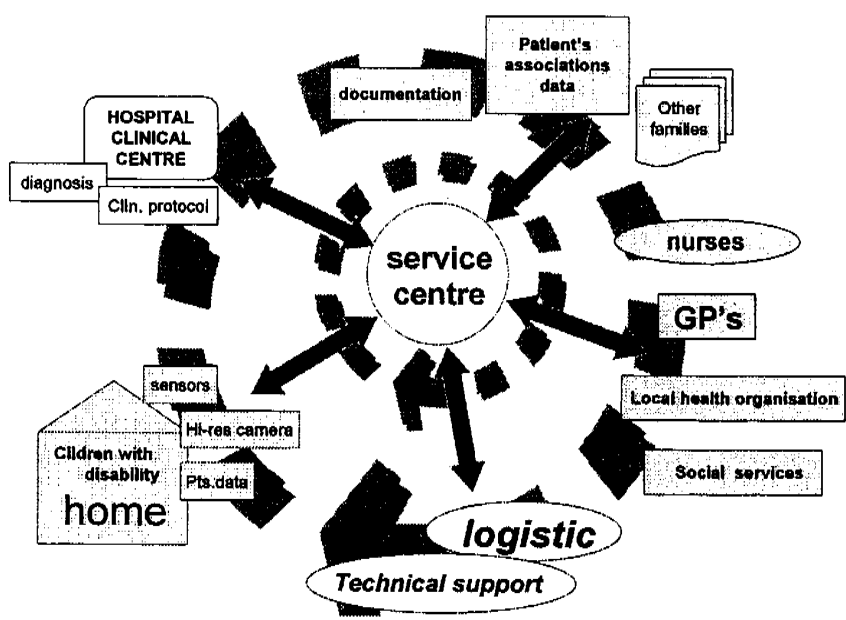

Figure 1: Actors involved in the project. 
The network allows the creation of a virtual community exchanging relevant information (education, family data, patient's associations data, database documentation, etc.). Doing this, Karma2 increases patient's quality of life, reduces the hospitalisation and assistance costs, and removes anxiety, distress and frustration from families making them feel inside an organized and efficient structure.

From the technological point of view, in the Karma2 project the assumption is that nowadays modern technologies are available, and the main effort must be directed toward their integration, accordingly to the specific needs of Home Care. In this communication, the focus is on the main technological elements of the project. In particular, special attention will be devoted to a medical device, developed within the project, that can be considered the starting element of the Karma2 infrastructure. In fact, it allows measurement of important physiological parameters that can be remotely controlled by the health care professionals, which can, on this basis, decide interventions and health service actions, involving the proper actors.

\section{Wearable device for measuring clinical data}

A block diagram of the main components of the medical device is reported in the following (Figure 2).

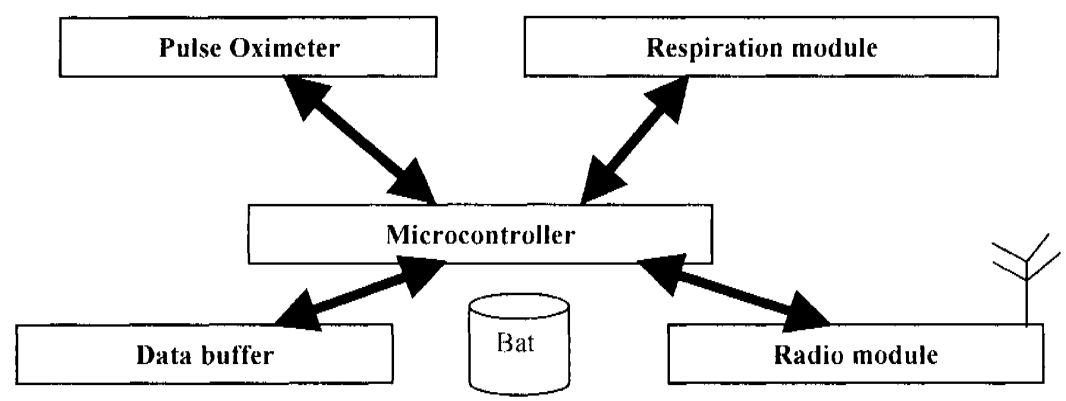

Figure 2: Block diagram of the warable device.

This medical device is able to measure:

1. Blood oxigen saturation;

2. Heart rate;

3. Respiration rate.

An important feature of this device is that it is wearable by the patient, which in this way is left free to move, and to walk, going on measuring the abovementioned parameters. This is already an advanced feature for a medical instrument, compared to many traditional instruments on the market. However, on the other hand many portable and also wearable devices already exist. What makes this device really innovative is the use of an advanced and quite new radio 
communication technology for transmitting measured data to a data collecting device inside the patient's house. This technology is called Bluetooth, and it will be briefly presented in the following. In fact, next sections will provide some details about some of the main components of the device.

\subsection{Pulse oximeter module}

The devices for detection of the blood oxygen saturation that, nowadays, are commonly used are the so-called pulse oximeters [3].

The great part of these devices is based on some key elements and principles. In a conventional pulse oximeter, a sensor probe is applied on a terminal part of the body (for instance, a finger, or an ear). This probe contains emitters of light in the red and infrared spectrum. The light passes through the vascular bed of the body part and is collected from some photo-detectors. Thus, a measurement is obtained of the red and infrared optical density. From the ratio between red and infrared optical density, the arterial oxygen saturation $(\mathrm{SpO} 2)$ value is determined based on empirical relationships.

The above-mentioned basic elements can be considered common to all the devices available on the market; what can change are some properties of the sensor probes (that is, the part of the device directly connected to the patient), such as the body part they are applied to, the shape, the duration of the probe itself.

The heart rate is easily recovered from the pulse oximeter as a secondary product of the oxygen saturation signal. In fact, this signal has variations connected to the heart beat. Thus, the time interval between two consecutive maxima reached in the saturation signal provides an estimation of the heart rate.

In our wearable device, we have realized the pulse oximetry function through integration of a commercial OEM Pulse Oximeter board (Nonin OEM II Module $^{\text {}}$ ). This OEM board is an advanced, quite new module, with little dimensions $(46 \times 34 \mathrm{~mm}$ ) with respect to other boards used in traditional Pulse Oximeter instruments.

The chosen module allows measurement of the oxygen saturation signal in the whole range $(0-100 \%)$, and of the heart rate in the $18-300$ beats per minutes range.

\subsection{Respiration rate module}

Many technologies are known for measuring the respiration rate. A common technique in the clinical practise, that can be used to detect the respiration rate, is capnography [4]. In fact, capnography provides measurements of carbon dioxide during the respiratory cycle, thus respiration rate can be easily derived. However, instruments for capnography require components that are not suitable for a wearable device.

Another possible technique is the impedance pneumography. Transthoracic impedance is determined by passing a current of a few microamperes, at a frequency of about $50 \mathrm{KHz}$, through the subject's thorax, and by measuring the 
voltage across the electrodes that generate the current itself. As the impedance depends on the thoracic volume, which varies during the respiration cycle, the breath rate can then be detected. The same electrodes that act as ECG leads can be used for impedance pneumography. This technique may be adequate for a wearable device, but, due to the needs of electrodes, it is not the most comfortable solution for the patient.

Another technique is based on the use of a thermistor, that is, a thin component placed by tape between the nose and the mouth, which detects the respiration flow, thus providing the respiration rate. Some sensors only monitor nasal airflow, wheras some other monitors both nasal and oral airflow. Similarly to previous technique, it is not the best choice for comfort and for cosmetic reasons.

A different technique exploits the piezoelectric sensor's capacity to produce a small voltage when a stress is applied to it. In this kind of system, a piezoelectric sensor is encased in a box, which has a plastic strip connected to it on both sides; then, a belt allows attaching the sensor to the patient. During breathing, the sensor is subjected to stress due to the expansion of the thorax or the abdomen, thus providing information on the respiration rate. The advantage of this technique is that the belt can be put over dresses and not directly over thorax, and on the other hand it can be hidden by dresses themselves. For these reasons, it is the technique that we have chosen for our project. This part of the project, however, is still under development, and some changes may be done as soon as actual testing on patients begins.

\subsection{Radio communication module: Bluetooth}

The Bluetooth protocol is nowadays the most promising technology for wireless point-to-point communications in an indoor environment. Bluetooth operates within the ISM band between 2.402-2.480 GHz, it is based on GFSK modulation and hopping over 79 channels, each displaced by $1 \mathrm{MHz}$, at 1600 hops per second, which make difficult to intercept Bluetooth signal. The data rate is $1 \mathrm{Mb} / \mathrm{s}$ in its original standard, and Bluetooth version 2.0 may reach 10 $\mathrm{Mb} / \mathrm{s}[5]$.

Bluetooth is adequate for short-range communications: in fact, it is made for communications within a so-called PAN, that is Personal Area Network (Figure 3). You can think to a PAN as a sort of bubble around the subjects, with 10 meters radius, which allows him to interact with all Bluetooth-enabled devices inside the bubble itself (such as mobile phones, printers, PDAs, etc.). 


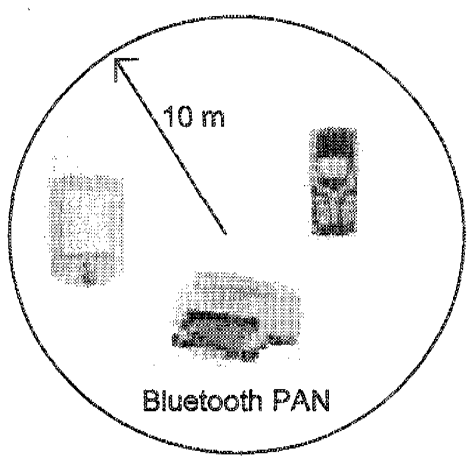

Figure 3: Bluetooth PAN with possible Bluetooth-enabled devices.

In some sense, Bluetooth can have applications similar to those typical of wireless infrared communications. However, the great advantage of Bluetooth compared to the latter is that, being a radio technology, it does not require to be in line of sight for transmission.

On the other hand, compared to other wireless, radio communication technologies, the first advantage of Bluetooth is lower consumption; thus, using Bluetooth makes sense for low power communications, whereas other technologies, such a WiFi, HomeRF, DECT, Hyperlan/2, may be more adequate in other cases. Indeed, these mentioned technologies are primarily device-toserver (i.e. LAN Access Point) technologies; conversely, Bluetooth is primarily a device-to-device (i.e. mobile phone to PDA) technology. It is also worth noting that Bluetooth has lower costs with respect to the other radio technologies. Despite of that, it is quite a complex and complete technology, also implementing security features such as authentication and encryption of transmitted data.

For all these reasons, Bluetooth was the best choice for our application. In fact, being included in a wearable device powered with batteries, it was mandatory to choose a wireless module with low consumption. On the other hand, we want the patient to be free of moving inside the house, going on measuring the physiological parameters and downloading them into the data collecting device, even if this latter is in another room (in the hypothesis, of course, that it is within Bluetooth range): for this reason, infrared technology could not be chosen. Finally, dealing with personal, clinical data, it was important to assure some security issues for transmission: Bluetooth was a good choice also from this point of view.

It is worth noting that, nowadays, several devices are already Blueoothenabled, and it seems that more and more will be in a near future. However, this is not the case in the field of medical devices. To our knowledge, really a few medical devices are Bluetooth-enabled so far. For this reason, we consider implementation of Bluetooth in our wearable device an important element of innovation. 
The basis of the Bluetooth functionality is obtained with a commercial device from ConnectBlue ${ }^{\circledR}$, which has been adequately specialized for our application through a special protocol, called $\mathrm{ACI}$ protocol.

\subsection{Microcontroller, data buffer and other components}

The microcontroller (family M16C form Mitsubishi ${ }^{(8)}$ ) manages all the presented modules. Although it is the core of the device, we do not provide many details here, since there are not special elements to be presented. Main functions of the microcontroller are managing the data acquisition, transferring measured data to the Bluetooth module, checking the level of the batteries. Moreover, if the patient is out of transmission for the Bluetooth module, and hence that data could not be collected from the data collecting device, the microcontroller temporarily stores the data into a data buffer (a Multimedia Card), until Bluetooth communication can occur again. It must be underlined that this is completely transparent to the user.

\section{The network: from the Web Phone to the Service Center}

The clinical data measured from the wearable device are downloaded via Bluetooth into a collecting device. This device is called the Web Phone. In fact, Web Phones are the remote, peripheral units of the project network, which connects all these devices to a Service Center, where a Web server for the project Web site, and a patients' database server are located (Figure 4).

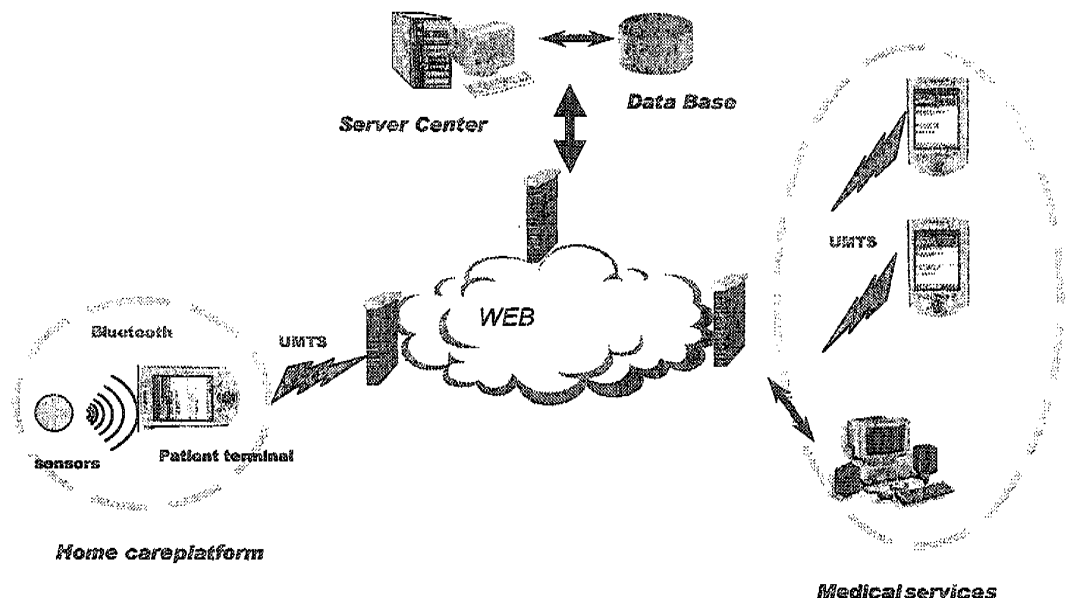

Figure 4: The project network.

The Web Phone has a double functionality:

1. it acts a "bridge" between the wearable device and the database server at the Service Center: it provides an Internet connection between these two units. 
2. it makes the Internet connection to the project Web site available for the patient: in this way, the patient and his family can get all the information of interest for them.

This second aspect has to be considered as important as the collection and control of the clinical data. In fact, accessing the project Web site can provide information about the various pathologies and diseases that a brain-injured child can have, and about the most recent findings in care, but also information on services available in the surroundings of the patients (physiotherapists, nurses, etc.). Moreover, the patients and families can have the possibility to chat, or to write emails, to other actors in the projects, and possibly to have real consultation with professionals, also through videoconferencing. All that can provide the feeling, very important for patients and families, to be within an organized and efficient virtual community tailored for their specific needs. Furthermore, some functionalities of the system are still under development and hence other services may be added in future.

From a technical point of view, the Web Phone is not a normal PC: in fact, we want the patient to have the chance to use it also when he is out of home: thus, a portable device was mandatory. The choice was a Webpad (or Tablet PC). The advantages of this type of device are:

1. it is light, and quite small (smaller not only than a PC, but also than a notebook);

2. it has however sufficient dimensions to provide quite a big screen (more, for instance, than a PDA).

The problem of the screen dimensions is important, since we have to provide the patients with an easy-to-use device, possibly also with touch screen functionality for easier surfing through the project Web site.

The Web Phone must be equipped with a Bluetooth module in order to interact with the wearable device, and with a GPRS module: in fact, one of the aim being maximum flexibility in using it, the Internet connection is obtained through GPRS, and hence there is no need to look for a telephone plug anymore. Moreover, as soon as it becomes available, we will use UMTS technology instead of GPRS. The use of UMTS is another element of innovation in the project: actually, with a data rate up to $2 \mathrm{Mbit} / \mathrm{s}$, also transmission of images or video streaming will become possible, with great benefit for the communication inside the project virtual community.

At the moment, we are still testing several Webpads and Tablet PCs, to select the best for our application: some of them have already GPRS or Bluetooth functionality integrated, but, to our knowledge, no one has already both. The missing functionality can be obtained with a PC Card or a CompactFlash Card (there are many Bluetooth card of this type, as well as GPRS card). Thus, the selected Web Phone must have at least one PC or CompactFlash slot.

It is worth noting that also the professionals involved in the project will be equipped with a sort of Web Phone, to get wireless and mobile access to the project Web site, and in particular to the patients' database, according to some proper levels of priviledge. For professionals, a PDA will be the right choice. 


\section{Conclusions and future developments}

The presented project has the aim to create an organized network for Home Care assistance of brain-injured children. The project, which is co-founded by the European Commission, contains several elements of innovation, both from a technical and organizational point of view. The project being currently in due course, some aspects have been already developed, but some other are still under study or under development. In particular, we are still working on an advanced system for managing information within the community. In fact, we are creating a system, based on the so-called Workflow and Knowledge Management techniques, able to establish the best flow of each specific piece of information generated within the project, from the actor that generates it to the actor that uses it. Finally, another central aspect of the project that will occur in the next months is the testing of the whole platform on actual patients, which will be provided by the clinical Partners of the Consortium. In a first phase, the system will be probably tested over 40 patients. Finally, it must be underlined that this organizational model for Home Care activities is, at the moment, tailored for a specific class of patients, but we believe in future it could be applied to other classes of patients with chronic diseases [6].

\section{References}

[1] Allen, A., et al., An Analysis of the Suitability of Home Health Care Visit for Telemedicine. J. of Telemedicine and Telecare, 5, pp. 90-96, 1999.

[2] Finkelstein, J., et al., Internet-based Home Asthma Telemonitoring, Chest, 117 (1), pp. 148-155, 2000.

[3] Middleton, P.M. \& Henry, J.A., Pulse Oximetry: Evolution and Direction. Int $J$ Clin Pract, 54, pp. 438-444, 2000.

[4] Anderson, C.T. \& Breen, P.H, Carbon Dioxide Kinetics and Capnography during Critical Care. Crit Care, 4, pp. 207-215, 2000.

[5] Bray, J., (ed). Bluetooth Application Developer's Guide, Syngress Media Inc: Rockland, 2001.

[6] Celler, B.G., Remote Monitoring of Health Status of the Elderly at Home. Int $J$ of Biomedical Computing, 40(2), pp. 147, 1995. 
